\title{
Dilated Fourth Ventricle in Arnold-Chiari Malformation Type II: Isolated Fourth Ventricle as Sequelae of Shunt?
}

- Case Report-

\author{
Shigetaka ANEGAWA, Takashi HAYASHI, Ryuichiro TORIGOE \\ and Tetsuzo OGASAWARA*
}

\author{
Departments of Neurosurgery and ${ }^{*}$ Neuroradiology, Institute of Neurosciences, \\ St. Mary's Hospital, Kurume, Fukuoka
}

\begin{abstract}
A female infant with Arnold-Chiari malformation type II developed cystic dilatation of the fourth ventricle at age 15 months. She received shunt emplacement into the dilated fourth ventricle to restore communication to the subarachnoid space and achieved improved symptoms and decreased ventricular size. Such dilatation is a typical feature of this malformation, and the isolation of the fourth ventricle is supposed to result from the cerebrospinal fluid shunting procedure. In this patient, the cause appeared to be external compression of the aqueduct by the beaking deformity of the mesencephalic spur, a characteristic of Arnold-Chiari malformation type 11.
\end{abstract}

Key words: myelomeningocele, isolated fourth ventricle, Arnold-Chiari malformation

\section{Introduction}

Isolated fourth ventricle manifests as a solitary dilatation of the fourth ventricle resulting from obstruction of the aqueduct of Sylvius, due to overdrainage by shunting procedures, or the foramina of Luschka and Magendie. ${ }^{3,5-7,10)}$ This condition may also result from hydrocephalus due to bacterial meningitis and intraventricular hemorrhage ${ }^{5,10)}$ Cystic dilatation of the fourth ventricle also occurs in patients with Arnold-Chiari malformation type II (ACM II). ${ }^{14)}$ Here, we describe clinical and neuroradiological findings in an ACM II patient and discuss the etiology of isolated fourth ventricle.

\section{Case Report}

A female infant with a diagnosis of fetal ventricular enlargement based on ultrasonic examination was delivered spontaneously after 41 weeks of gestation. The Apgar score was 8 at 1 minute after birth, and the birth weight was $3009 \mathrm{~g}$ with $33.6 \mathrm{~cm}$ head circumference. A myelomeningocele in the lumbosacral region was found and repaired the same day. At 2

Received June 25, 1992; Accepted January 7, 1993 weeks of age, a ventriculoperitoneal shunt was emplaced, requiring three subsequent revisions because of shunt malfunction. Even though the shunt functioned excellently, she developed progressive respiratory failure. Magnetic resonance (MR) images demonstrated hypoplasia of the corpus callosum, caudal displacement of the fourth ventricle, and medullary kinking at the $\mathrm{C} 2$ level (Fig. 1). C1-3 laminectomies including suboccipital craniectomy and dural plasty of the posterior fossa were performed. The postoperative course was uneventful and the brainstem symptoms were relieved. However, swallowing dysfunction, spasticity, and respiratory distress progressed again, so re-examination was needed.

On admission, at the age of 15 months, she was generally in poor condition. Neurological examination demonstrated paraplegia below the L5 level, weak gag reflexes bilaterally, poor suck, and fasciculations of the tongue. Poor tone was noted in the sternocleidomastoid muscle. Computed tomographic (CT) scans showed a marked dilatation of the fourth ventricle without evidence of dilatation of the third and lateral ventricles (Fig. 2). Repeat MR images demonstrated cystic dilatation of the fourth ventricle compressing the pons anteriorly and 


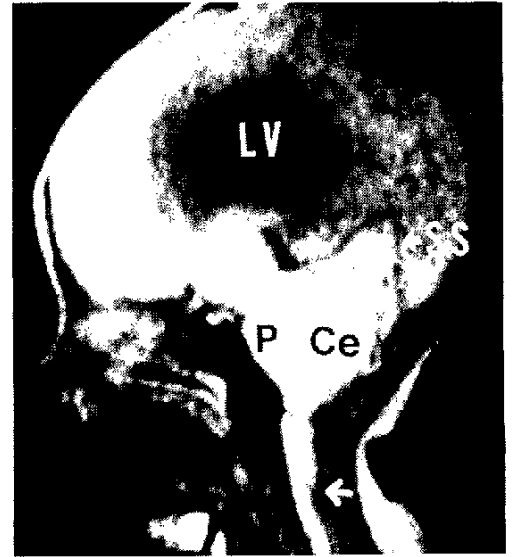

Fig. $1 T_{1}$-weighted $M R$ image $(0.2 T)$, showing no dilatation of the fourth ventricle. Note the medullary kinking (arrow). Ce: cerebellum, LV: lateral ventricle, $P$ : pons, SS: straight sinus.

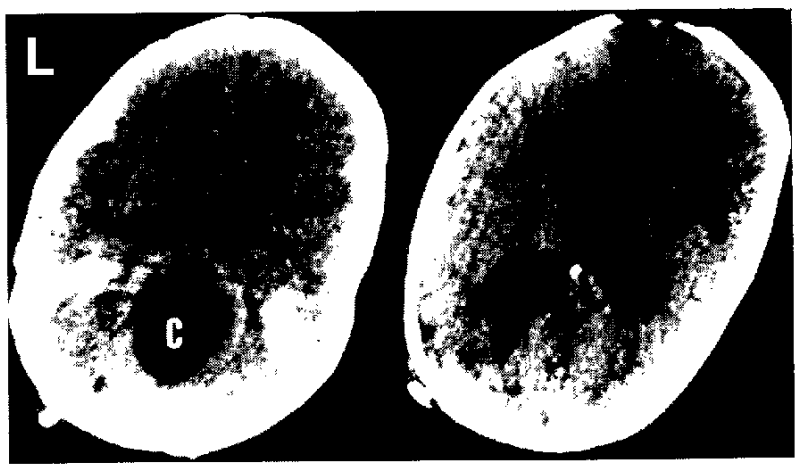

Fig. 2 CT scans at the age of 15 months, demonstrating enlargement of the fourth ventricle (C), while the lateral ventricle is almost normal.

cerebellum posteriorly with caudal displacement of the brainstem (Fig. 3). Communication of the aqueduct of Sylvius was obstructed by the mesencephalic spur and the caudal part of the fourth ventricle protruded into the upper cervical canal.

A linear vertical scalp incision was made with her in the prone position. Opening the dura mater demonstrated the bilateral tonsils covered by the thick fibrous membrane herniating through the foramen magnum. The fibrous band was removed under the surgical microscope, visualizing a thin cyst wall. On opening the cyst wall, cerebrospinal fluid was discharged. A shunt tube was emplaced from the fourth ventricle to the subarachnoid space around the cerebellar tonsil enlarged in the previous
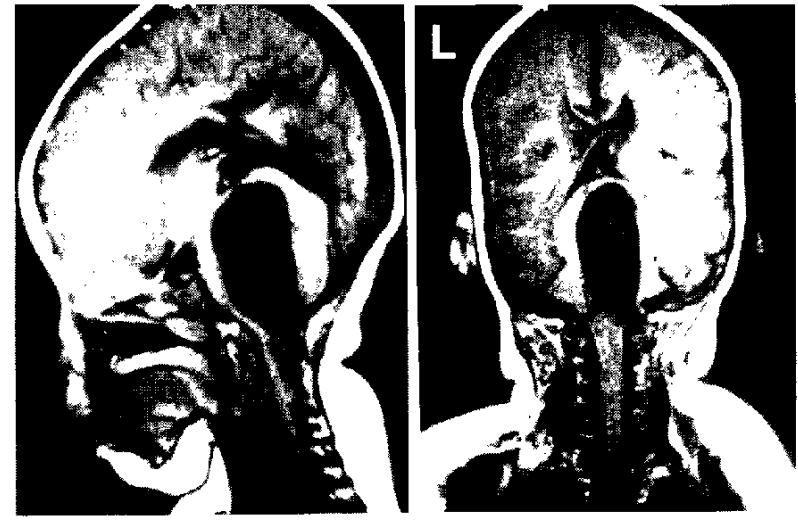

Fig. $3 \mathrm{~T}_{1}$-weighted MR images (1.5 T), sagittal (left) and coronal views (right), demonstrating cystic dilatation of the fourth ventricle and syringomyelia. Note the characteristic mesencephalic spur and non-visualization of the aqueduct of Sylvius.

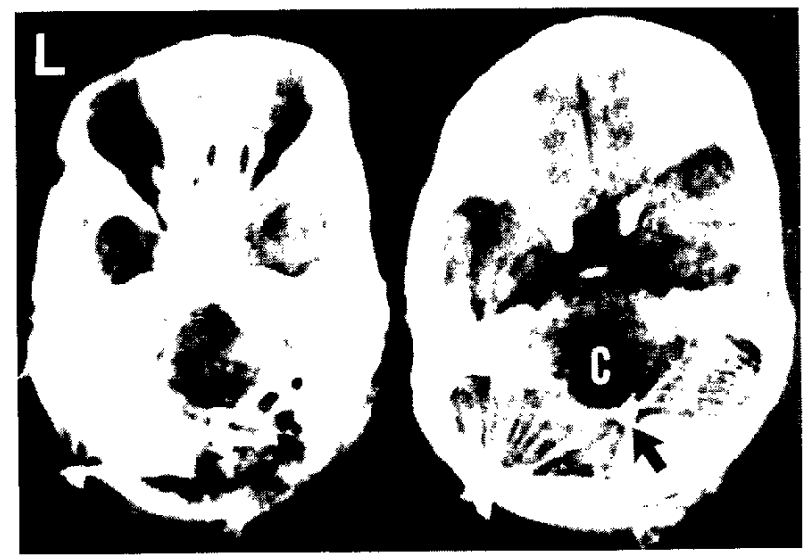

Fig. 4 CT scans 1 day after cyst-subarachnoid shunting, showing a marked decrease in the size of the fourth ventricle (C). Arrow indicates the shunt tube.

operation.

The postoperative course was uneventful and the lower cranial nerve paresis was relieved. Repeat CT scans showed that the size of the fourth ventricle had decreased (Fig. 4). However, some symptoms persisted, probably due to the tethering effect of the lower cranial nerves secondary to the caudal displacement of the brainstem and the upper cervical cord.

\section{Discussion}

The fourth ventricle in ACM II generally appears as a long sagittally flattened cavity with nearly parallel 
walls and no obvious lateral recess, twice as long as normal, and extending into the cervical spinal canal..$^{5,11-13)}$ In contrast, Zimmerman et al. ${ }^{15)}$ and Naidich et al. ${ }^{91}$ reported that the fourth ventricle was small or nearly normal except for a small number of patients with a slightly enlarged fourth ventricle. Venes et al. ${ }^{14)}$ reported three types of fourth ventricle anomalies including cystic dilatation in ACM II patients with shunts. They also reported two patients whose fourth ventricle was initially normal and then enlarged.

Isolated fourth ventricle is defined as a cystic dilatation of the fourth ventricle caused by obstruction both inlet and outlet of the fourth ventricle. ${ }^{3,5-7,10)}$ This phenomenon is caused by veil obstruction originating in stenosis ${ }^{6)}$ or sequelae of cerebrospinal fluid overdrainage resulting in functional obstruction of the aqueduct with reactive inflammation due to hemorrhage, meningitis, or surgical insult. ${ }^{10)}$

In our case, the fourth ventricle was initially small and elongated. However, based on the decrease in the size of the lateral ventricle, cystic dilatation of the fourth ventricle occurred without overdrainage. MR imaging showed that the proximal wall of the dilated fourth ventricle consisted of beaking deformity of the tectum increasing with dilatation of the fourth ventricle. The beaking deformity of the tectum (mesencephalic spur) is a characteristic of ACM II. ${ }^{1,2,4)}$ Adeloye $^{1)}$ reported that the length of the spur increased linearly with age. Russell and Donald ${ }^{(3)}$ reported that all ACM II patients except one had communicating type hydrocephalus. Moreover, Cameron ${ }^{21}$ reported that the aqueduct was abnormally narrow in $50 \%$ of patients and the most frequent cause was inflammation. True congenital obstruction occurred in only one of 20 cases. Therefore, the aqueduct in ACM II patients is thought to be patent though narrow in most cases.

The etiology of cystic dilatation of the fourth ventricle cannot be derived from clinical findings, but serial MR observation provides a possible explanation. Patients demonstrating cystic fourth ventricle ${ }^{14)}$ manifested delayed symptoms from the posterior fossa after the initial operation as in our case. Furthermore, in our case, the lateral ventricles were not slit but of normal size, suggesting that the inflammation due to operation and functional obstruction due to slit ventricle cannot be responsible for the obstruction of the aqueduct. Also, the dilated fourth ventricle shrank immediately after the shunt restored communication to the subarachnoid space. Therefore, the dilatation of the fourth ventricle is not secondary to the ACM Il but due to disorder of the cerebrospinal fluid circulation. Masters ${ }^{81}$ indicated that the aqueductal stenosis was secondary to the external compression of the mesencephalon. Presumably aqueductal obstruction may be caused by the growth of the mesencephalic spur with age and ultimately compresses the originally narrow aqueduct. Therefore, isolated fourth ventricle in ACM II is caused by external compression by the mesencephalic spur and/or enlarged inferior horn of the lateral ventricle but not veil obstruction or inflammatory occlusion.

ACM II patients presenting with symptoms from the posterior fossa with MR imaging showing evidence of cystic dilatation of the fourth ventricle should receive shunt emplacement to restore communication to the subarachnoid space, allowing immediate relief of symptoms.

\section{References}

1) Adeloye A: Mesencephalic spur (beaking deformity of the tectum) in Arnold-Chiari malformation. $J$ Neurosurg 45: 315-320, 1976

2) Cameron AH: The Arnold-Chiari and other neuroanatomical malformations associated with spina bifida. J Path Bact 73: 195-211, 1957

3) Couture A, Baud C, Veyrac C, Frerebeau PH, Bonnet $\mathrm{H}$ : Isolated fourth ventricle: Ultrasonic diagnosis and follow-up. Ann Radiol (Paris) 29: 345-352, 1986

4) Daniel PM, Strich SJ: Some observations on the congenital deformity of the central nervous system known as the Arnold-Chiari malformation. $J$ Neuropath Exp Neurol 17: 255-266, 1958

5) Eller TW, Pasternak JF: Isolated ventricles following intraventricular hemorrhage. $J$ Neurosurg 62: 357362,1985

6) Foltz EL, Defeo DR: Double compartment hydrocephalus: A new clinical entity. Neurosurgery 7: $551-559,1980$

7) Hawkins JC III, Hoffman HJ, Humphreys RP: Isolated fourth ventricle as a complication of ventricular shunting. $J$ Neurosurg 49: 910-913, 1978

8) Masters CL: Pathogenesis of the Arnold-Chiari malformation: The significance of hydrocephalus and aqueduct stenosis. J Neuropathol Exp Neurol 37: 56-74, 1978

9) Naidich TP, Pudlowski RM, Naidich JB: Computed tomographic signs of the Chiari II malformation. III: Ventricles and cisterns. Radiology 134: 657-663, 1980

10) Oi S, Matsumoto S: Slit ventricles as a cause of isolated ventricles after shunting. Childs Nerv Sysi 1: 189-193, 1985

11) Peach B: Cystic prolongation of fourth ventricle. An anomaly associated with the Arnold-Chiari malformation. Arch Neurol (Chicago) 11: 609-612, 1964

12) Peach B: Arnold-Chiari malformation. Anatomic features of 20 cases. Arch Neurol 12: 613-621, 1965 
13) Russell DS, Donald C: The mechanism of internal hydrocephalus in spina bifida. Brain 58: 203-215, 1935

14) Venes JL, Black KL, Latack JT: Preoperative evaluation and surgical management of the Arnold-Chiari II malformation. $J$ Neurosurg 64: 363-370, 1986

15) Zimmerman RD, Breckbill D, Dennis MW, Davis $\mathrm{RO}$ : Cranial CT findings in patients with meningo- myelocele. AJR 132: 623-629, 1979

Address reprint requests to: S. Anegawa, M.D., Department of Neurosurgery, Institute of Neurosciences, St. Mary's Hospital, 422 Tsubukuhon-machi, Kurume, Fukuoka 830, Japan. 